\title{
The circadian pattern of cardiac autonomic modulation in a middle-aged population
}

\author{
Xian Li $\cdot$ Michele L. Shaffer $\cdot$ Sol Rodriguez-Colon • \\ Fan He $\cdot$ Deborah L. Wolbrette $\cdot$ Peter Alagona Jr • \\ Chuntao Wu - Duanping Liao
}

Received: 3 June 2010/ Accepted: 14 December 2010/Published online: 15 January 2011

(C) The Author(s) 2011. This article is published with open access at Springerlink.com

\begin{abstract}
Objectives To examine the circadian pattern of cardiac autonomic modulation (CAM) and its attributes in general population.

Methods We obtained 24-h beat-to-beat RR data using a high resolution 12-lead Holter ECG in a communitydwelling sample of 115 non-smokers. We performed heart rate variability (HRV) analysis on the normal RRs from each 5-min segment to obtain time-specific HRV indices: high (HF; 0.15-0.40 Hz) and low (LF; 0.04-0.15 Hz) frequency powers, standard deviation of $R R$ intervals (SDNN), and the square root of the mean of the sum of the squared differences of the adjacent RR intervals (RMSSD). For each individual, we fit the segment-specific HRV data to a cosine periodic function, and estimated 3 individuallevel cosine function parameters to quantify the circadian variation: the mean $(M)$, amplitude $(A)$, and acrophase $(\theta)$. We then used a random-effects meta-analysis to summarize the $M, A$, and $\theta$, and their $95 \%$ confidence intervals (CI). Results The mean age was 56 (SD 8) years, with $63 \%$ female and $76 \%$ white. The averages of $M, A$ and $\theta$
\end{abstract}

X. Li · M. L. Shaffer - S. Rodriguez-Colon · F. He · C. Wu ·

D. Liao $(\square)$

Department of Public Health Sciences,

Penn State University College of Medicine,

600 Centerview Dr. Suite 2200, A210, Hershey, PA 17033, USA

e-mail: dliao@psu.edu

X. Li

Cardiovascular Institute and Fu Wai Hospital,

Chinese Academy of Medical Sciences,

Peking Union Medical College, Beijing, China

D. L. Wolbrette · P. Alagona Jr

Department of Medicine, Division of Cardiology,

Penn State University College of Medicine, Hershey, PA, USA
(95\%CI) of $\log \mathrm{HF}$ were $3.59(3.43-3.76) \mathrm{ms}^{2}, 0.61$ (0.54-0.68) $\mathrm{ms}^{2}$, and 3:10 (2:25-3:55) AM, respectively, and that of RMSSD were 22.3 (20.5-24.1) ms, 6.5 (5.4-7.5) ms, 3:45 (2:55-4:35) AM, respectively. Older age is associated with lower mean of HRV. Males have higher oscillation amplitude than females. The acrophase of LF/HF was earlier in females than in males, and in younger individuals than in older individuals.

Conclusions The circadian pattern of CAM can be quantified by 3 cosine parameters of HRV, which are correlated with age and gender.

Keywords Autonomic modulation - Heart rate variability $\cdot$ Periodic rhythm $\cdot$ Community population

\section{Introduction}

The cardiac rhythm is regulated or modulated by sympathovagal balance. Heart rate variability (HRV) is a commonly used noninvasive measurement of cardiac autonomic modulation (CAM) [1-6]. Several studies in patients have shown that lower HRV calculated from shortterm normal RR intervals was a strong and independent predictor of mortality after an acute myocardial infarction (MI) [7-9] and sudden cardiac death [10]. It has been reported in prospective population-based studies that lower HRV calculated from short-term normal RR intervals, ranging from several minutes to several hours, is associated with significantly higher risk of mortality $[11,12]$ and incidence of coronary heart disease [13, 14]. It has been recognized from previous studies that autonomic modulation fluctuates on a 24-h basis like a biological clock, or exhibits a circadian rhythm [15-18]. For example, Bonnemeier et al. reported the differences of mean HRV at 
different times of the day, particularly the night-time and day-time differences in HRV. None of these previous studies of the fluctuation of HRV provided the quantitative normative data of the nonlinear fluctuation of HRV over the entire 24-h time.

Although it has been proposed to use more quantitative approaches, such as cosine periodic regression models, to detail the mathematical properties of the normal CAM circadian variation over $24 \mathrm{~h} \mathrm{[19]} \mathrm{in} \mathrm{order} \mathrm{to} \mathrm{better}$ understand the effects of various conditions on CAM, such as chronic disease, medication, and "jet-lag", very few studies have used cosine periodic regression to quantify the overall 24-h circadian pattern of HRV. A cosine periodic regression curve contains three major parameters: the mean $(M)$, amplitude $(A)$, and acrophase $(\theta)$, where $M$ measures the overall average of a HRV index, which is similar to the mean HRV used in previous literature [1-18], $A$ measures the amplitude of the oscillation of an HRV index, and $\theta$ measures the clock time when the highest oscillation (amplitude) is reached. Nakagawa et al. [20] reported the cosine-shaped circadian pattern of HRV in a small group $(n=20)$ of young adult volunteers. Massin et al. [21] reported the cosine pattern of HRV in a group of children. However, both studies used the group-level time-specific averages of the HRV index to calculate the cosine function parameters, which did not account for between-subjects variability. As a result, these studies cannot provide the reference ranges of these cosine parameters, e.g. 95\% confidence intervals (CIs). In fact, it is possible that the between-subjects variation of $\mathrm{HRV}$ is larger than the within-subject variation over time, which can only be analyzed using an approach that accounts for both between- and within-subject variations. Thus, the objective of this study is to obtain the quantitative measures of the HRV circadian pattern and their normative ranges in a middle-aged community-dwelling sample, specifically the normative ranges of the three cosine regression parameters - the mean $(M)$, amplitude $(A)$, and acrophase $(\theta)$. A secondary objective is to examine the influence of age and gender on the circadian patterns of HRV in this population.

\section{Population and methods}

Population

The data collected for the Air Pollution and Cardiac Risk and its Time Course (APACR) study are used for this report. The APACR study, funded by NIEHS (1 R01 ES014010), was previously described in detail [22]. Briefly, all study participants were recruited from community-dwelling individuals residing in central Pennsylvania, mostly from the Harrisburg Metropolitan area. The inclusion criteria for the APACR study included age $\geq 45$ years, not smoking, and not having severe cardiac problems (defined as diagnosed valvular heart disease, congenital heart disease, acute myocardial infarction or stroke within 6 months, or congestive heart failure). We enrolled and examined 115 individuals for the APACR study. All study participants, after providing written informed consent, were examined between November, 2007 and June, 2009.

Study participants were examined in the Penn State College of Medicine General Clinical Research Center (GCRC) in the morning between 8 and $10 \mathrm{AM}$. All participants fasted for at least $8 \mathrm{~h}$ before the clinical examination. After completing a health history questionnaire, a trained research nurse measured seated blood pressure three times, height, and weight, and drew $50 \mathrm{ml}$ of blood for blood biomarker assays according to the blood sample preparation protocols. A trained investigator hooked up the Holter ECG recorders between 9 and 10 AM. Participants were given an hourly activity log to record special events that occurred in the next $24 \mathrm{~h}$. Participants were then released to go on with their regular daily routines in the period of ongoing Holter recording. The next morning the participants came back to the GCRC to unhook the Holter monitors and return the completed activity log. Penn State University College of Medicine IRB approved the study protocol.

\section{Continuous Holter ECG recording}

A high fidelity (sampling frequency $1,000 \mathrm{~Hz}$ ) 12-lead H-Scribe Holter System (Mortara Instrument, Inc., WI, USA) was used for 24-h beat-to-beat ECG data collection. The high fidelity ECG significantly increases the resolution and enhances the accuracy of various wave form measurements. The standardized operation procedures for the APACR study developed by the study investigators were followed rigorously in the data collection, retrieval, and offline processing. The main objective of the offline processing was to verify the Holter-identified ECG waves, and to identify and label additional electronic artifacts and arrhythmic beats in the ECG recording. After removing artifacts and ectopy beats with standardized visional inspection, we obtained beat-to-beat normal RR interval data for HRV analysis.

\section{Heart rate variability}

The entire 24-h normal beat-to-beat RR interval data were divided into 5-min segment RR data. Thus, each individual provided 288 segments of 5-min RR data. The RR data for HRV analysis were processed according to current recommendations [1]. Within each segment, any RR interval 
$<400 \mathrm{~ms},>2,000 \mathrm{~ms}$, or where the ratio from two adjacent RR intervals was $<0.80$ or $>1.20$ were excluded from the HRV analysis. The time- and frequency-domain HRV analyses were performed on the remaining normal RR interval data if the total length of such normal RR intervals was $>4$ min ( $80 \%$ of original data), using a software program (HRV Analysis Software; Department of Physics, University of Kuopio, Finland). When performing frequency-domain HRV analysis, we used fast Fourier transformation (FFT). Briefly, the adjacent RR interval data were interpolated using a piecewise cubic spline interpolation approach, with a $2 \mathrm{~Hz}$ sampling rate. The FFT was performed on the equidistantly interpolated RR time series. We used a second order polynomial model to remove the slow non-stationary trends of the HRV signal. The following HRV indices were calculated as measures of CAM: standard deviation of all RR intervals (SDNN, ms), square root of the mean of the sum of the squares of differences between adjacent RR intervals (RMSSD, ms), power in the high frequency range $(0.15-0.40 \mathrm{~Hz}, \mathrm{HF})$, power in the low frequency range $(0.04-0.15 \mathrm{~Hz}, \mathrm{LF})$, and the ratio of LF to $\mathrm{HF}(\mathrm{LF} / \mathrm{HF})$. Power in the very low frequency range $(\leq 0.04 \mathrm{~Hz})$ was not studied here because of the shortduration of the window used (5 min) [1]. Following current recommendations [1], we performed logarithmic transformations on HF and LF prior to statistical analysis.

\section{Statistical analysis}

From the 115 individuals, we excluded 20 individuals from this report because of the following reason(s): technical problems with the Holter recording $(n=1)$, insufficient normal RR intervals for HRV analysis $(<20 \mathrm{~h}$ of ECG data) $(n=2)$, a history of diabetes $(n=9)$, and a history of cardiovascular disease (CVD, $n=8$ ). As a result, this report used the data from the remaining 95 individuals. Each individual contributed up to 288 segments of 5-min RR interval data within $24 \mathrm{~h}$, resulting in up to 27,360 data segments. We analyzed 26,714 segments of HRV data after excluding segments with less than 4 min of normal RR interval data.

We used a two-stage modeling approach to achieve the objectives of this study. In the first stage, we fit one cosine periodic regression model for each individual to get the individual-level parameters $(M, A$, and $\theta)$ of the cosine curves as the indicators of nonlinear fluctuation of HRV. Specifically, in the first stage, we fit each HRV variable based on all available 5-min segments within $24 \mathrm{~h}$ from each participant to a cosine periodic regression model [23] using nonlinear least squares: $\operatorname{HRV}_{i}(t)=M_{i}+A_{i} \cdot \cos [$ $\left.2 \pi \cdot\left(t-\theta_{i}\right) / T\right]+\varepsilon_{i}, i=1, \ldots, 95$, in which $M_{i}$ is the daily average of HRV of the $i$ th subject, $A_{i}$ is the fluctuation amplitude of HRV of the $i$ th subject around $M_{i}, t$ is the time-specific segment order number, $T$ is the total number of 5-min segments in $24 \mathrm{~h}, \theta_{i}$ is the acrophase (the lag from the reference time point ( $9 \mathrm{AM})$ to the time of the zenith of the cosine curve fit to the data of the $i$ th subject), and $\varepsilon_{i}$ is the error term of the $i$ th subject. One unit of $t$ corresponds to $5 \mathrm{~min}$, with 1 indicating 9:00 AM to 9:05 AM, 2 indicating 9:05 AM to 9:10 AM... etc. In the second stage, we used random-effects meta-analysis to obtain the population estimates of $M, A$ and $\theta$, and their $95 \%$ CIs [24] from the individual cosine parameters obtained from the first stage to achieve the first objective of this study. Unlike a fixed-effects meta-analysis, which would account for only within-subject variability, the random-effects meta-analysis allows for both within- and between-subject variability. The random-effects meta-analysis method was also used to test $A=0$ (zero amplitude test) and assess the impact of the population attributes, such as age and sex, on the three cosine parameters of the HRV variables (the secondary objective). To visualize the circadian pattern of each HRV variable, we plotted the two-stage model predicted circadian pattern of each HRV variable over the 24-h clock time, with the corresponding time-specific segment average of the HRV variable superimposed. The time-specific segment averages are derived from a linear mixed-effects model [25], where time is treated as a fixed, categorical variable and the within-subject covariance matrix has a first-order autoregressive structure. This model places no specific functional form on the relationships between the HRV variables and time. All analyses were performed using SAS version 9.1 software (SAS Institute Inc., Cary, NC, USA).

\section{Results}

The demographic data, CVD risk factors, and the HRV indices of the entire study population are shown in Table 1 . The mean age of the participants was 56 (SD 8) years, with $63 \%$ female, $76 \%$ non-Hispanic white, and $24 \%$ minorities (including Blacks, Hispanics, and Chinese). The data showed that their general health and chronic CVD risk factors are similar to that of the middle-aged American general population.

The normative range of circadian HRV—-first objective

The estimates of the three cosine periodic regression parameters and their 95\% CIs for each HRV variable are presented in Table 2. The acrophases of $\log \mathrm{HF}$ and RMSSD (both are reflective of the vagal modulation) were very similar-the point estimates are around 3:30 AM \pm 20 min. The acrophases of $\log$ LF and SDNN (both are reflective of sympathetic and vagal modulation) also were 
Table 1 The study population characteristics

\begin{tabular}{|c|c|c|c|c|c|}
\hline & Total & $\begin{array}{l}\text { Male } \\
(n=35)\end{array}$ & $\begin{array}{l}\text { Female } \\
(n=60)\end{array}$ & $\begin{array}{l}\text { Non-Hispanic } \\
\text { white }(n=72)\end{array}$ & $\begin{array}{l}\text { Non-white } \\
(n=23)\end{array}$ \\
\hline Age (years) & $56.5(8.1)$ & $54.6(7.7)$ & $57.5(8.1)$ & $57.7(8.1)$ & $52.6(6.5)$ \\
\hline Body mass index $\left(\mathrm{kg} / \mathrm{m}^{2}\right)$ & $26.8(4.9)$ & $26.8(3.3)$ & $26.8(5.7)$ & $26.4(4.9)$ & $28.2(4.8)$ \\
\hline Systolic blood pressure $(\mathrm{mmHg})$ & $121.7(15.1)$ & $122.9(15.4)$ & $121.0(15.0)$ & $122.5(15.1)$ & $119.1(15.1)$ \\
\hline Diastolic blood pressure $(\mathrm{mmHg})$ & $75.0(8.9)$ & $76.9(9.1)$ & $73.9(8.7)$ & $74.9(8.5)$ & $75.3(10.4)$ \\
\hline Fasting blood glucose (mg/dl) & $84.8(10.6)$ & $88.9(11.1)$ & $82.5(9.6)$ & $83.3(9.6)$ & $89.6(12.4)$ \\
\hline Total cholesterol (mg/dl) & $206(36)$ & $206(34)$ & $208(37)$ & $206(31)$ & $204(50)$ \\
\hline High density lipoprotein cholesterol (mg/dl) & $56.9(16.8)$ & $45.5(12.1)$ & $63.4(15.7)$ & $59.5(17.3)$ & $48.6(12.1)$ \\
\hline Low density lipoprotein cholesterol (mg/dl) & $124.5(31.9)$ & $125.9(31.1)$ & $123.7(32.6)$ & $124.6(28.4)$ & $124.3(42.1)$ \\
\hline Triglyceride (mg/dl) & $120.4(77.6)$ & $151.2(96.6)$ & $102.9(58.5)$ & 109.8 (71.8) & $155.0(87.1)$ \\
\hline \multicolumn{6}{|l|}{ HRV variables ${ }^{\mathrm{a}}$} \\
\hline Ln HF $\left(\mathrm{ms}^{2}\right)$ & $3.6(0.8)$ & $3.5(0.7)$ & $3.7(0.9)$ & $3.6(0.7)$ & $3.7(1.0)$ \\
\hline $\operatorname{Ln} \operatorname{LF}\left(\mathrm{ms}^{2}\right)$ & $4.8(0.7)$ & $4.9(0.5)$ & $4.7(0.8)$ & $4.8(0.7)$ & $4.6(1.0)$ \\
\hline $\mathrm{LF} / \mathrm{HF}$ ratio & $4.7(2.1)$ & $5.6(2.2)$ & $4.2(1.8)$ & $5.0(2.2)$ & $3.7(1.0)$ \\
\hline SDNN (ms) & $43.3(13.3)$ & $44.1(12.5)$ & $42.8(13.8)$ & $43.5(12.4)$ & $42.8(16.4)$ \\
\hline RMSSD (ms) & $22.3(8.7)$ & $21.4(8.5)$ & $22.9(8.9)$ & $22.1(8.5)$ & $23.3(10.0)$ \\
\hline Heart rate (beats/min) & $75.8(9.7)$ & $75.9(11.0)$ & $75.7(8.9)$ & $75.0(9.0)$ & $77.6(11.3)$ \\
\hline
\end{tabular}

Continuous variables are presented as mean (SD); categorical variables are presented as percentage (\%)

${ }^{\text {a }}$ Overall average of the intra-subject means of 5-min segments

Table 2 The parameter estimates (and 95\% CI) of the cosine periodic regression model of HRV indices

\begin{tabular}{|c|c|c|c|}
\hline & Mean $(95 \% \mathrm{CI})$ & Amplitude* $(95 \% \mathrm{CI})$ & Acrophase $(95 \% \mathrm{CI})^{\mathrm{a}}$ \\
\hline $\log \mathrm{HF}\left(\mathrm{ms}^{2}\right)$ & $3.59(3.43-3.76)$ & $0.61(0.54-0.68)$ & $3: 10(2: 25-3: 55) \mathrm{AM}$ \\
\hline $\log \operatorname{LF}\left(\mathrm{ms}^{2}\right)$ & $4.75(4.60-4.91)$ & $0.44(0.39-0.49)$ & 4:55 (3:50-6:00) AM \\
\hline SDNN (ms) & $43.2(40.5-45.9)$ & $9.4(8.1-10.7)$ & $5: 10(4: 20-5: 55) \mathrm{AM}$ \\
\hline RMSSD (ms) & $22.3(20.5-24.1)$ & $6.5(5.4-7.5)$ & $3: 45(2: 55-4: 35) \mathrm{AM}$ \\
\hline $\mathrm{LF} / \mathrm{HF}$ ratio & $4.68(4.26-5.10)$ & $1.35(1.15-1.56)$ & $3: 30(2: 20-4: 35) \mathrm{PM}$ \\
\hline Heart rate (bpm) & $75.8(73.8-77.8)$ & $10.8(9.9-11.7)$ & $2: 15(1: 55-2: 35) \mathrm{PM}$ \\
\hline
\end{tabular}

$* p<0.0001$ for zero amplitude tests of all HRV indices

${ }^{\text {a }}$ Calculated as the unit to zenith $\pm 2 \mathrm{SE}$, and translated into clock time

very similar-the point estimates are around 5:00 $\mathrm{AM} \pm$ $10 \mathrm{~min}$. The acrophase of $\mathrm{LF} / \mathrm{HF}$ ratio was $3: 30$ in the afternoon, and that of heart rate was 2:15 in the afternoon. The tests of zero amplitude were highly significant $(p<0.0001)$ for every HRV variable, clearly suggesting a significant circadian variation. The circadian variation of HRV indices are graphically presented in Fig. 1, overlaying the time-specific segment averages and the periodic curve fit from the point estimates of the three cosine periodic regression parameters calculated from the random-effects meta-analysis. These plots show a distinctive day-night variation on all HRV variables analyzed on a 24-h basis.
Comparisons of cosine parameters of HRV by population attributes-secondary objective

The association between each cosine parameter and participant's age, ethnicity, and gender are presented in Table 3, as the regression coefficients $(\beta)$, standard errors (SE), and $p$ values. These were calculated from the random-effects meta-analysis regression models. The between gender comparison of the $M s$ in Table 3 indicate that there is no significant gender difference, except for LF/HF ratio, where $M$ is significantly higher in males than in females. For between gender comparisons of the $A s$, males have higher fluctuation amplitude of $\log \mathrm{HF}(p=0.08), \log \mathrm{LF}$ 

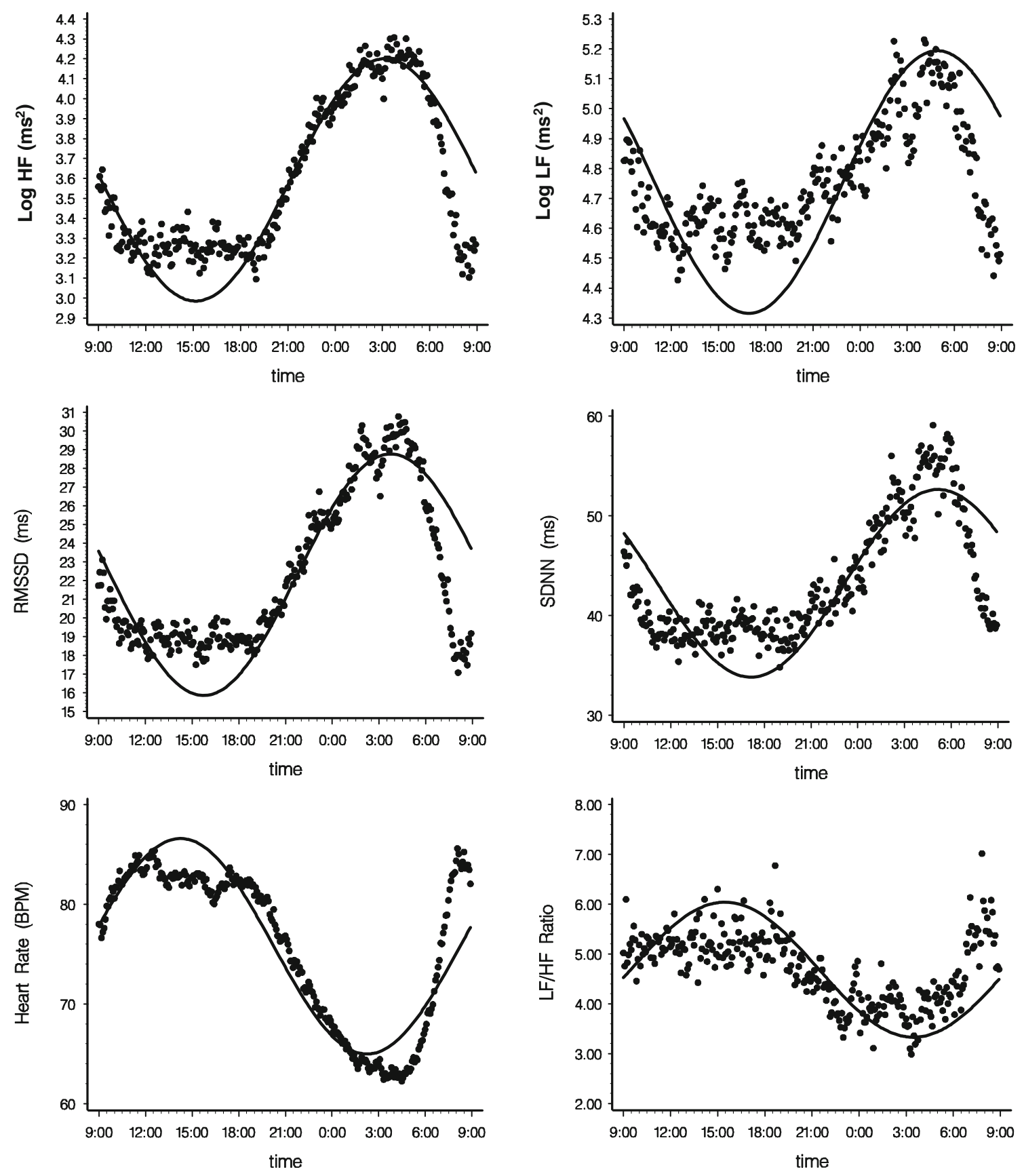

Fig. 1 The circadian periodic curves of HRV variables overlaid with time-specific segment averages. Overall periodic curve derived from random-effects meta-analysis (Solid line). Time-specific segment average values (Dots)

$(p=0.01), \operatorname{SDNN}(p=0.04)$, and LF/HF ratio $(p=0.04)$ than females. The between gender comparison of the $\theta s$ in Table 3 indicate that there is no significant gender difference, except for LF/HF ratio, where the $\theta$ in males is significantly delayed for almost $3 \mathrm{~h}(p=0.01)$. Meanwhile, older age is significantly associated with lower $M$ for $\log$ HF $(p<0.01), \log$ LF $(p<0.01)$, SDNN $(p<0.05)$, and RMSSD $(p<0.05)$. Age is not significantly associated with the $A$ of any HRV index, except for the LF/HF ratio, where older age is associated with significantly lower $A(p=0.04)$. Interestingly, older age is significantly associated with delayed $\theta$ in RMSSD (approximately $1 \mathrm{~h}, p<0.05$ ) and LF/HF ratio (approximately $2 \mathrm{~h}, p<0.01$ ). Few of the ethnic differences in these three cosine function parameters are statistically significant. The very few that are statistically significant or marginally significant, namely LF, SDNN, LF/HF ratio, and HR, the non-Hispanic white showed a similar pattern to the male gender in these data. 
Table 3 The regression coefficient $(\beta)$ estimates and SE of major population attributes on mean, amplitude, and acrophase of the circadian periodic curves of HRV from random-effects meta-analysis

\begin{tabular}{|c|c|c|c|c|c|c|c|c|c|}
\hline & \multicolumn{3}{|l|}{ Male } & \multicolumn{3}{|c|}{ Age (per 10 years) ${ }^{\mathrm{a}}$} & \multicolumn{3}{|c|}{ Non-Hispanic white } \\
\hline & $\beta$ & SE & $p$ value & $\beta$ & SE & $p$ value & $\beta$ & SE & $p$ value \\
\hline \multicolumn{10}{|c|}{$\log \mathrm{HF}\left(\mathrm{ms}^{2}\right)$} \\
\hline$M^{\mathrm{b}}$ & -0.30 & 0.17 & 0.08 & -0.33 & 0.10 & 0.002 & -0.06 & 0.19 & 0.74 \\
\hline$A^{\mathrm{b}}$ & 0.13 & 0.07 & 0.08 & -0.03 & 0.04 & 0.55 & 0.002 & 0.08 & 0.98 \\
\hline$\theta^{\mathrm{b}}$ & -3.7 & 9.5 & 0.70 & 3.9 & 5.9 & 0.51 & -12.3 & 10.9 & 0.26 \\
\hline \multicolumn{10}{|c|}{$\log \operatorname{LF}\left(\mathrm{ms}^{2}\right)$} \\
\hline$M$ & 0.11 & 0.16 & 0.46 & -0.29 & 0.10 & 0.003 & 0.31 & 0.18 & 0.09 \\
\hline$A$ & 0.14 & 0.05 & 0.01 & 0.02 & 0.03 & 0.57 & 0.13 & 0.06 & 0.03 \\
\hline$\theta$ & -20.8 & 13.1 & 0.12 & -14.3 & 8.2 & 0.08 & -30.5 & 15.3 & 0.05 \\
\hline \multicolumn{10}{|c|}{ SDNN (ms) } \\
\hline$M$ & 0.41 & 2.82 & 0.88 & -4.40 & 1.75 & 0.01 & 2.99 & 3.25 & 0.36 \\
\hline$A$ & 2.78 & 1.35 & 0.04 & -0.21 & 0.83 & 0.80 & 2.71 & 1.55 & 0.08 \\
\hline$\theta$ & -18.6 & 10.1 & 0.07 & -9.4 & 6.2 & 0.13 & -5.4 & 11.6 & 0.64 \\
\hline \multicolumn{10}{|c|}{ RMSSD (ms) } \\
\hline$M$ & -2.27 & 1.86 & 0.22 & -2.57 & 1.15 & 0.03 & -0.21 & 2.14 & 0.92 \\
\hline$A$ & 1.30 & 1.08 & 0.23 & -0.73 & 0.67 & 0.28 & 0.58 & 1.24 & 0.64 \\
\hline$\theta$ & 2.1 & 10.5 & 0.84 & 14.1 & 6.5 & 0.03 & -8.5 & 12.1 & 0.49 \\
\hline \multicolumn{10}{|c|}{$\mathrm{LF} / \mathrm{HF}$ ratio } \\
\hline$M$ & 1.66 & 0.39 & $<0.001$ & 0.06 & 0.24 & 0.79 & 1.54 & 0.45 & 0.001 \\
\hline$A$ & 0.43 & 0.21 & 0.04 & -0.27 & 0.13 & 0.04 & 0.18 & 0.23 & 0.44 \\
\hline$\theta$ & 35.0 & 13.2 & 0.01 & 22.2 & 8.2 & 0.008 & 7.3 & 15.1 & 0.63 \\
\hline \multicolumn{10}{|c|}{ Heart rate $(\mathrm{bpm})$} \\
\hline$M$ & -0.5 & 2.1 & 0.80 & -1.8 & 1.3 & 0.16 & -1.6 & 2.4 & 0.50 \\
\hline$A$ & 0.7 & 1.0 & 0.44 & -0.3 & 0.6 & 0.61 & 1.3 & 1.1 & 0.24 \\
\hline$\theta$ & 4.6 & 3.7 & 0.22 & -0.001 & 2.3 & 1.00 & -8.4 & 4.3 & 0.05 \\
\hline
\end{tabular}

${ }^{a}$ Age was analyzed as a continuous variable; thus, the regression coefficients correspond to the changes for every 10 -year increase in age

${ }^{\mathrm{b}} M, A$, and $\theta$ : parameters of the cosine periodic curvēmean, amplitude, and acrophase; 1 unit of $\theta$ corresponds to 5 min, with a positive $\theta$ of 1 unit indicating 5 min later, a positive $\theta$ of 6 units indicating 30 min later, 12 units indicating $1 \mathrm{~h}$ later, and so on

\section{Discussion}

Our first objective in this study was to quantitatively describe the circadian pattern of CAM using cosine periodic regression models and to obtain the normative range of these cosine parameters. To this aim, the data presented in Table 2 and Fig. 1 demonstrate the circadian patterns, which can be appropriately captured by these three cosine function parameters. In general, higher values of HF, LF, RMSSD, and SDNN are indicative of more parasympathetic modulation. Overall, these HRV variables follow a very similar circadian pattern-decreasing during the AM hours and reaching the lowest levels in the afternoon, and turning upwards in the evening and reaching the acrophase in the early morning 3:00-5:00, and finally starting to decline again after the acrophase. On the other hand, HR and $\mathrm{LF} / \mathrm{HF}$, where higher values are indicative of more sympathetic modulation, showed an opposite diurnal pattern-increasing during the AM hours and reaching the highest levels in the afternoon 2:00-4:00, then turning downwards after that and reaching the lowest points after midnight before the early morning hours, and finally starting to increase again after the lowest points. Most importantly, the tests of zero amplitude were highly statistically significant (all $p<0.0001$ ) for every HRV variable analyzed. These highly significant $p$ values suggest a significant day-night circadian variation. The point estimates of the three cosine parameters of $\log \mathrm{HF}$ and LF/HF from our data and from Nakagawa et al. [20] are similar, except that they did not involve time-domain HRV and did not observe a significant circadian variation of LF.

We only found two previous studies in healthy individuals that examined the circadian pattern of HRV as a cosine function-one involved 20 healthy young adult volunteers [20] and another was based on 57 children [21]. Compared to these two previous studies, the current study has several strengths. This study used a two-stage analytic approach, which represents an important improvement over 
previous studies. Specifically, this two-stage modeling approach allowed us to first capture within individual variation of HRV over time for each participant and allowed for between individual differences in the circadian variation, which in turn was accounted for in the final stage of obtaining study sample-level cosine parameters and their 95\% CIs. In contrast, previous studies calculated the timespecific averages from the sample, and used such averages to estimate the cosine parameters. Such an approach is computationally efficient in estimating the average of the cosine parameters, but it eliminated the between-subjects differences in HRV on any time point, and as a consequence, it cannot provide the $95 \% \mathrm{CI}$ of the cosine parameters. For the same reason, the cosine parameters estimated from the previous approach cannot be easily analyzed in terms of the impact of population attributes. Other factors such as physical activity, obesity, and cardiac co-morbidity are known to have an adverse impact on cardiac autonomic modulation. As illustrated in the associations between age, ethnicity, sex, and HRV circadian pattern in this study (secondary objective), the two-stage approach we used can be used to effectively compare the effects of various factors on the circadian pattern of HRV.

Furthermore, this report is based on a communitydwelling sample of healthy middle-aged individuals, which is a traditional "high risk" group for acute cardiac events, such as myocardial infarction and sudden cardiac death. As such, our HRV circadian pattern data can be generalized to this important age group. Previous studies sampled much younger individuals, and were based on smaller sample sizes [20, 21].

A secondary objective of this study was to examine the influences of population attributes, such as gender, age, and ethnicity, on the three cosine regression parameters. For the between gender comparisons of the cosine parameters of HRV, our data suggest that there is no significant gender difference in the $M s$ of HRV curves, except for LF/HF ratio, where the $M$ is significantly higher in males than in females, indicative of a higher sympathetic and lower parasympathetic modulation in males than in females (Table 3). We did not find significant gender difference for the $M s$ of HF, LF, SDNN, and RMSSD in our sample. This is consistent with previous reports of the disappearance of gender differences of mean HRV at approximately 50 years of age [26-28]. For the comparisons of the As of HRV curves, males have higher amplitude than females on almost every HRV variable analyzed (Table 3). To our knowledge, this is the first study to report that males have higher fluctuation amplitude of HRV than females. For the comparisons of the $\theta s$ of HRV curves, there is no significant gender difference in the acrophase time, except for $\mathrm{LF} / \mathrm{HF}$ ratio, where the acrophase is significantly delayed for almost $3 \mathrm{~h}(p=0.01)$ in males. The association between age and the three cosine parameters in our data is very consistent with the literature [18, 26-28]: in general, older age is significantly associated with lower mean levels, lower amplitude, and later acrophase time of HRV. These findings confirm the adverse age effects on the balance of parasympathetic and sympathetic modulation of heart beats. In our data, there were very few nonconsistent associations between white and non-white groups. This may be due to the "non-white" population in our sample consisting of one African American, eleven Chinese, and eleven Hispanics. We did not have a large enough sample size to compare white with each of the individual ethnicities. Others have reported that persons with angina [23], type 2 diabetes, and elevated blood pressure [29] had impaired circadian pattern of HRV. Having established the effects of age and gender on the circadian parameters of HRV, future studies are needed to quantify the effects of these chronic conditions on the circadian parameters of HRV, and to investigate beneficial effects on the circadian parameters of HRV from physical activity or pharmacological treatment of various chronic conditions.

We excluded individuals with a history of CVD or diabetes from this report, which is consistent with our primary objective- to obtain the normal circadian pattern of HRV in an apparently healthy middle-aged population. As a consequence, the generalizability of our data to individuals with such chronic conditions may be limited. Similarly, we only included nonsmokers as study participants. Thus, the results may not be applicable to smokers. Lastly, the ECG data were collected via Holter recorder. As such, individuals were carrying on their regular routines, including any habitual physical activity, which is known to influence CAM [30].

\section{Conclusions}

In this community-based sample of middle-aged individuals, the HRV variables, as measures of the balance of autonomic modulation, exhibit a distinct circadian rhythm over the 24-h period. The HRV circadian rhythm follows a cosine periodic function, which can be quantified with three parameters-the mean, the amplitude, and the acrophase time. The three cosine function parameters together with their respective $95 \%$ CIs estimated from our data can be used as normal ranges of 5-min period time- and frequency-domain HRV circadian variations. Population attributes, such as gender and age, are associated with the HRV cosine circadian parameters.

Acknowledgment National Institute of Environmental Health Sciences (1 R01 ES014010) funded this study. 


\section{Conflicts of interest Nothing to disclose.}

Open Access This article is distributed under the terms of the Creative Commons Attribution Noncommercial License which permits any noncommercial use, distribution, and reproduction in any medium, provided the original author(s) and source are credited.

\section{References}

1. Task Force of the European Society of Cardiology, the North American Society of Pacing and Electrophysiology (1996) Heart rate variability: standards of measurement, physiological interpretation and clinical use. Circulation 93:1043-1065

2. Akselrod S, Gordon D, Ubel FA, Shannon DC, Berger AC, Cohen RJ (1981) Power spectrum analysis of heart rate fluctuation: a quantitative probe of beat-to-beat cardiovascular control. Science 213:220-222

3. Pomeranz B, Macaulay RJ, Caudill MA et al (1985) Assessment of autonomic function in humans by heart rate spectral analysis. Am J Physiol 248:H151-H153

4. Pagani M, Lombardi F, Guzzetti S et al (1986) Power spectral analysis of heart rate and arterial pressure variabilities as a marker of sympatho-vagal interaction in man and conscious dog. Circ Res 59:178-193

5. Ewing DJ, Neilson JM, Travis P (1984) New method for assessing cardiac parasympathetic activity using 24 hour electrocardiograms. Br Heart J 52:396-402

6. Malik M, Camm AJ (1990) Heart rate variability. Clin Cardiol 13:570-576

7. Kleiger RE, Miller JP, Bigger JTJ, Moss AJ, the Multicenter Post-Infarction Research Group (1987) Decreased heart rate variability and its association with increased mortality after acute myocardial infarction. Am J Cardiol 59:256-262

8. Malik M, Farrell T, Cripps T, Camm AJ (1989) Heart rate variability in relation to prognosis after myocardial infarction: selection of optimal processing techniques. Eur Heart $\mathrm{J}$ 10:1060-1074

9. Bigger JT, Fleiss JL, Steinman RC, Rolnitzky LM, Kleiger RE, Rottman JN (1992) Frequency domain measures of heart period variability and mortality after myocardial infarction. Circulation 85:164-171

10. La Rovere MT, Pinna GD, Maestri R et al (2003) Short-term heart rate variability strongly predicts sudden cardiac death in chronic heart failure patients. Circulation 107:565-570

11. Dekker JM, Crow RS, Folsom AR et al (2000) Low heart rate variability in a 2-minute rhythm strip predicts risk of coronary heart disease and mortality from several causes: the ARIC Study: atherosclerosis risk in communities. Circulation 102:1239-1244

12. Tsuji H, Venditti FJJ, Manders ES et al (1994) Reduced heart rate variability and mortality risk in an elderly cohort. The Framingham Heart Study. Circulation 90:878-883

13. Liao D, Cai J, Rosamond WD et al (1997) Cardiac autonomic function and incident coronary heart disease: a population-based case-cohort study. The ARIC study. Am J Epidemiol 145:696706

14. Tsuji H, Larson MG, Venditti FJ Jr et al (1996) Impact of reduced heart rate variability on risk for cardiac events. Framingham Heart Study. Circulation 94:2850-2855

15. Huikuri HV, Kessler KM, Terracall E, Castellanos A, Linnaluoto MK, Myerburg RJ (1990) Reproducibility and circadian rhythm of heart rate variability in healthy subjects. Am J Cardiol 65:391-393

16. Furlan R, Guzzetti S, Crivellaro W et al (1990) Continuous 24-hour assessment of the neural regulation of systemic arterial pressure and RR variabilities in ambulant subjects. Circulation 81:537-547

17. Molgaard H, Sorensen KE, Bjerregaard P (1991) Circadian variation and influence of risk factors on heart rate variability in healthy subjects. Am J Cardiol 68:777-784

18. Bonnemeier H, Richardt G, Potratz J et al (2003) Circadian profile of cardiac autonomic nervous modulation in healthy subjects: differing effects of aging and gender on heart rate variability. J Cardiovasc Electrophysiol 14:791-799

19. Minors DS, Waterhouse JM (1988) Mathematical and statistical analysis of circadian rhythms. Psychoneuroendocrinology 13:443-464

20. Nakagawa M, Iwao T, Ishida S et al (1998) Circadian rhythm of the signal averaged electrocardiogram and its relation to heart rate variability in healthy subjects. Heart 79:493-496

21. Massin MM, Maeyns K, Withofs N, Ravet F, Gerard P (2000) Circadian rhythm of heart rate and heart rate variability. Arch Dis Child 83:179-182

22. Liao D, Shaffer ML, Rodriguez-Colon S et al (2010) Adverse effects of fine particulate air pollution on ventricular repolarization. Environ Health Perspect 118:1010-1015

23. D'Negri CE, Marelich L, Vigo D et al (2005) Circadian periodicity of heart rate variability in hospitalized angor patients. Clin Auton Res 15:223-232

24. DerSimonian R, Laird N (1986) Meta-analysis in clinical trials. Control Clin Trials 7:177-188

25. Laird NM, Ware JH (1982) Random-effects models for longitudinal data. Biometrics 38:963-974

26. Yamasaki Y, Kodama M, Matsuhisa M et al (1996) Diurnal heart rate variability in healthy subjects: effects of aging and sex difference. Am J Physiol 271 (1 Pt 2):H303-H310

27. Umetani K, Singer DH, McCraty R, Atkinson M (1998) Twentyfour hour time domain heart rate variability and heart rate: relations to age and gender over nine decades. J Am Coll Cardiol 31:593-601

28. Kuo TB, Lin T, Yang CC, Li CL, Chen CF, Chou P (1999) Effect of aging on gender differences in neural control of heart rate. Am J Physiol 277:2233-2239

29. Kondo K, Matsubara T, Nakamura J, Hotta N (2002) Characteristic patterns of circadian variation in plasma catecholamine levels, blood pressure and heart rate variability in Type 2 diabetic patients. Diabet Med 19:359-365

30. Bernardi L, Valle F, Coco M, Calciati A, Sleight P (1996) Physical activity influences heart rate variability and very-lowfrequency components in Holter electrocardiograms. Cardiovasc Res 32:234-237 